\title{
CMS Virtual Visits: Engaging Audiences Worldwide In Conversation About Cutting Edge Science
}

\author{
Cecilia Uribe Estrada (on behalf of the CMS Collaboration) ${ }^{a, *}$ \\ ${ }^{a}$ Benemérita Universidad Autónoma de Puebla, Puebla, Mexico \\ E-mail: cecilia.uribe.estrada@cern.ch
}

Advances in information and communications technologies (ICTs) have given rise to innovative uses of web-based video tools for global communication, enhancing the impact of large research facilities, including their outreach and education programmes. As an example, the CMS Virtual Visits programme launched by the CMS Collaboration at CERN uses videoconferencing to communicate with schools and other members of the public around the globe.

The goal of the programme is to break down geographical barriers and allow more people to enter the world of science, physics and particle physics. CMS Virtual Visits offer students, teachers and the general public a unique opportunity to explore the CMS detector. Through a web-based videoconference, CMS scientists interact with "remote" visitors in their native language, explain the physics and technology behind the CMS detector, and answer their questions.

Since September 2014, more than 35,000 people from around the world have participated in CMS Virtual Visits. We present an overview of our experience, feedback collected from participants and discuss potential development for the future.

40th International Conference on High Energy physics - ICHEP2020

July 28 - August 6, 2020

Prague, Czech Republic (virtual meeting)

\footnotetext{
${ }^{*}$ Speaker
} 


\section{Introduction}

CERN is home to the Large Hadron Collider (LHC), the most energetic particle accelerator in the world and a leading centre in High Energy Physics (HEP) research. CERN has a vast outreach programme, supported by its different experiments and groups, that makes science more accessible to the general public. With the discovery of the Higgs Boson in 2012 [1], which led to the 2013 Nobel Prize for Physics, more and more people worldwide have turned their attention to CERN and to the experiments that reported the discovery: ATLAS [2] and CMS [3].

As new advances in information and communications technologies (ICTs) are developed, CERN has incorporated innovative tools such as web-based video for global communication. The CMS Virtual Visits programme launched by the CMS Collaboration at CERN uses videoconferencing to communicate with schools, communities and the general public worldwide and is one of the most successful programmes in the outreach activities at CERN.

This paper presents the fundamentals of the CMS Virtual Visits programme and its impact in connecting researchers, students, teachers and the general public with cutting edge science from CERN.

\section{Virtual Visits}

A Virtual Visit is a unique opportunity to explore the detector live with scientists from around the world acting as guides. Audiences are mainly students, teachers and the general public. These connections are possible thanks to the increased availability of videoconferencing systems offering new opportunities and innovative practices that are emerging in classrooms. Virtual Visits allow the participants to get remote access to CERN's facilities and learn how a major scientific collaboration, such as CMS, built and operates such a state of the art detector [4]. On the 6th of October 2003 a pilot Virtual Visit took place at CERN, with 200 students from schools around Ireland [5]. This pilot visit formed the basis to establish Virtual Visits [6] as an important education and outreach project, with the main goal of bringing science to students and the general public around the globe, as well as opening a conversation with researchers in cutting edge experiments.

Each year, up to 45,000 people visit the ATLAS and CMS experimental sites in person [7]. Virtual Visits offer this experience to thousands around the globe who are unable to come to the laboratory in person. The first CMS virtual visit took place in the autumn of 2011 with students from Oveges High School in Hungary. Thanks to a technical stop, the scientists guiding the visit were able to get access to the cavern and showed the students attending the visit the CMS detector in all its splendour, $100 \mathrm{~m}$ below ground. Since then, more and more participants, mainly from schools, were offered the opportunity to visit the CMS experiment virtually [8]. Virtual Visits were officially launched in September 2014 [9]. To this day more than 35,000 people from around the world have participated in CMS Virtual Visits.

Figure 1 shows the number of participants per year since 2014. The largest numbers of visits were registered in 2015 and 2016. It is interesting to note that in 2015 the collision energy was increased to $13 \mathrm{TeV}$ (with the start of Run 2), the largest ever recorded in a particle accelerator. The decrease in participation in 2019 was due to a limit of 4 visits per month. It is worth noting that a planned three-year shutdown from 2019 to 2021, known as Long Shutdown 2 (LS2), during 
which maintenance and upgrades are carried out, allows the detector to be prepared for the high luminosity phase.

Figure 2 shows the distribution of the main reasons for CMS Virtual Visits. The largest share, at $55 \%$, is due to school events. The second largest contribution, with almost $30 \%$, is related to Open Days or public events organised by CERN or other institutions. University events constitute nearly $10 \%$ of the total, and exhibitions and science centres come close to $3 \%$, with the remainder going to teachers, workshops and other functions. It is noteworthy that more than $50 \%$ of the type of audiences participating in Virtual Visits is composed of high school students. This is important as one of the main goals of the visits is to increase interest among young students in Science, Technology, Engineering and Mathematics (STEM).

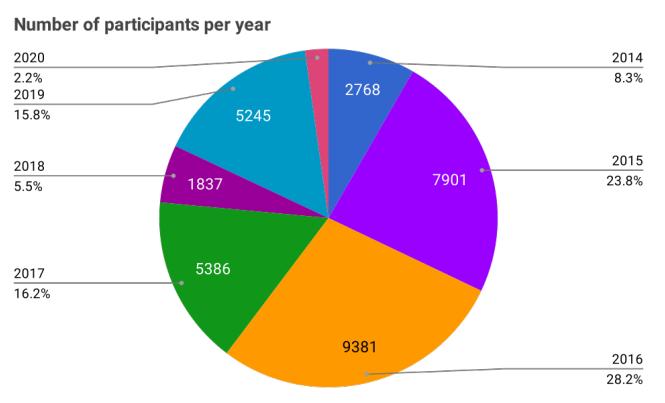

Figure 1: Diagram showing the number of participants per year during CMS Virtual Visits since 2014, when they were launched officially. The two largest percentages incidentally coincide with the increase in collision energy. The small percentage in 2020 is related to the COVID-19 pandemic and its effects, which greatly perturbed normal activities.

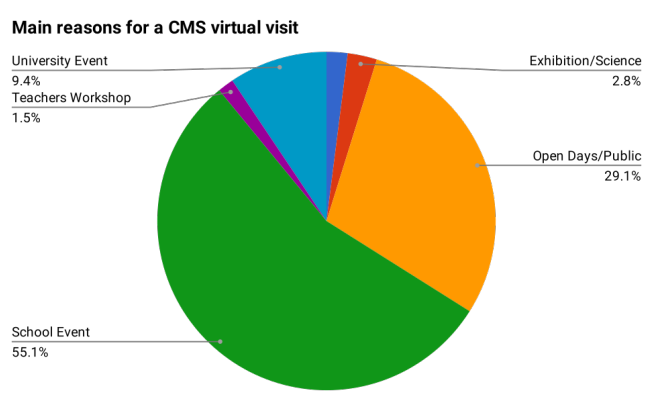

Figure 2: Diagram showing the percentage of the main reasons for a CMS Virtual Visit. School events comprise the largest percentage at $55 \%$, with high school students making up the largest share of audiences of the visits. The general public makes up around $30 \%$ of the participation, with close to $12 \%$ due to university students and the rest to teachers.

\section{Technology Employed}

Having access to the CERN communication infrastructure allows CMS to reach audiences around the world in an effective and cost efficient way. The connections use a combination of webbased video-conferencing [10], webcasting and audio-video hardware. The technical requirements for the remote locations are quite straightforward and consist of a computer with a minimum network speed of $1 \mathrm{Mbps}$, a video projector/TV screen, as well as a microphone and videoconferencing system that is simple to install and run. Other software such as Vidyo, Skype, Zoom, Google Meet, Google Hangouts, etc. can be used. The latter has been used for the "Hangout With CERN" series [11].

\section{Organisation of the Virtual Visit}

Virtual Visits are organised to provide meaningful experiences to the different types of participating audiences. It is crucial to take into account the different languages, ages and educational 
backgrounds of the participants. It is common to have more than one school or audience attending the Virtual Visit at the same time, provided that the language is the same for all. Matching requests to have guides who speak different languages is possible thanks to the size of the CMS Collaboration, which has around 3,600 international members.

The CMS outreach group helps with the organisation of the Virtual Visits and provides the technical team and equipment at CERN. Volunteers from the CMS collaboration may act as hosts at the CERN site or as contacts in remote locations, although other teachers or organisers may play this role as well. Around one week before the Virtual Visit takes place, the technical team at CERN will connect to the venue, making sure that there is a good connection and the Virtual Visit will go as planned. This is important as it may help preempt some issues such as sound quality, image, etc. that could otherwise compromise the visit.

The training of new volunteers from CMS is achieved through a shadow-shift during a preceding visit or by co-hosting a visit with one of the more experienced hosts. A list of volunteers is kept to match possible guides with future requests of Virtual Visits and outreach activities. Many of the guides include scientists, students, engineers, etc. who are very active and volunteer in other outreach activities.

During the Virtual Visit at CERN, besides the technical team, it is convenient to have at least two guides: one on the surface, and another showing the detector (in the event access is granted). This allows the guide on the surface to talk about the CMS experiment while the other guides the public, using a helmet camera, through the different corridors, tunnels and elevator until reaching the detector 100m underground. Communication can switch back and forth as needed between the two guides, including the case in which the signal is lost during the $100 \mathrm{~m}$ descent. This model has been very successful as the audience is always engaged with the visit.

At the venue there is at least one scientist or coordinator in charge. It is advised that the coordinator gives an introduction to the CMS Experiment before the Virtual Visit starts. This allows the public to come up with questions which can be answered by the guides at CERN.

Virtual Visits are recorded and kept on a permanent site for future reference. Feedback is collected at the end of the event at the venue and sent to CERN for future improvements.

\section{Feedback on Virtual Visits}

Outreach activities aim at inspiring young people to ask questions and get involved in science. CMS scientists participate willingly in different events organised around the year by CERN, such as the European Researchers' Night held the 28th of September 2018. On that night the Virtual Visits attracted around 1,000 students, parents, teachers and members of the general public from Hungary, Italy, Lithuania, Mexico and Portugal [12]. Figure 3 shows CMS scientists from Mexico and Colombia at CERN, guiding the audience during the Virtual Visit at the University of Sonora in Mexico.

The connections are scheduled at different times to allow the audiences to explore the facilities comfortably and talk to the researchers in their native tongue, including obtaining answers to questions they may have. The feedback was overwhelmingly positive and the researchers, all of whom actively engaged the audience throughout the visits, received great applause. Some of the comments at the venues were: 


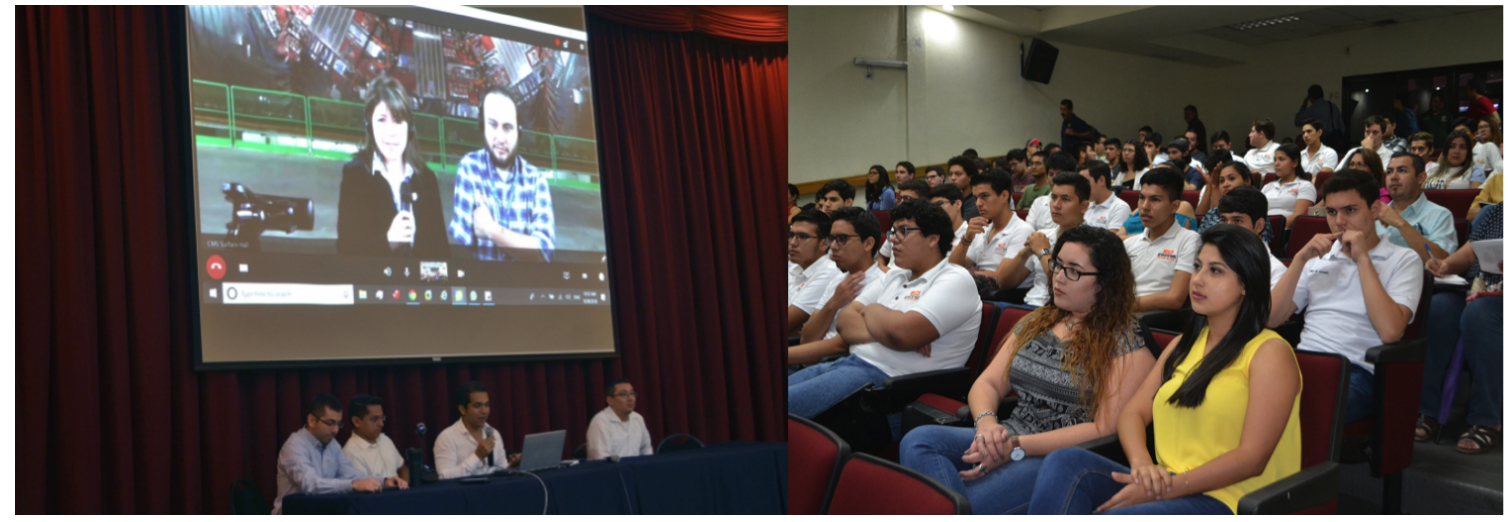

Figure 3: CMS researchers Cecilia Uribe Estrada and Jose Andres Monroy, guiding the Virtual Visit at CERN during the Researchers' Night with the audience at the University of Sonora, Mexico.

Egle Gaule, Head of Department of Research and Innovations in Kaunas University of Technology in Lithuania, was astonished by this innovative way of visiting real lab facilities. She also noted the importance of the fact that "the data obtained from the CMS Experiment is accessible to the public, allowing anyone who is interested in it to test their 'forces' in calculating the results of collisions of accelerated particles and possibly discovering the Higgs boson".

Alfredo M. Castaneda Hernandez, physics professor from the University of Sonora, Mexico: "I hope in the future we will have the opportunity to continue offering CMS Virtual Visits, not only here in the University of Sonora, Hermosillo, but all around Mexico and other countries to inspire young students".

Some other comments expressed by high school students at the Preparatoria Emiliano Zapata of the Benemérita Universidad Autónoma de Puebla (BUAP) Mexico after another Virtual Visit were:

Ali: "The Virtual Visit was like right out of the movies! Kind of 'Angels and Demons'! It made me realise that physics goes far beyond Newton and now I am much more curious to understand more about physics!"

Linda: "It was very interesting, they showed us that physics is not just what we learn in class but that it has many other applications in areas such as medicine, engineering and practically all the technology that we use! I really liked the connection with medicine as I want to pursue a career in the health sector!"

Jorge: "It was very cool to be transported to a different location in the world without leaving our school and to realise that there are so many varied and important technologies which we can have access to!"

\section{Partnerships}

Having international partnerships helps in reaching more students and the general public around the world, making frontier science available to a wider audience. At CERN, different experiments such as ATLAS and CMS organise Virtual Visits to reach broader audiences. Although there are 
some differences in the organisation of their visits, the main goal is the same: connect the public with frontier research being developed at CERN [13].

In addition, a partnership has recently been formed between International Masterclasses [14], QuarkNet and the International Particle Physics Outreach Group (IPPOG). These three organisations implemented the first large, entirely online Masterclass called BAMC (Big Analysis of Muons in CMS) during the week of the 13th of April 2020. By all measures this event was a resounding success with the participation of 23 international schools and 4 countries (China, Mexico, UK, USA). Over 230 students analysed 11,633 CMS events in $\sim 2$ days, and 180 students and teachers attended a webinar to discuss results and a CMS lecture during that week. The interest in April was such that a similar event was organised in May with 460 students worldwide analysing 23,541 events [15]. BAMC was designed for students and teachers working online. A simplified CMS masterclass analysis was planned with online support from teachers and CMS physicists so students could perform the measurements from home.

As a further example, CMS scientists joined forces during the COVID-19 pandemic to keep students active, organising webinars to talk about HEP. On June 12th, 2020 the Benemérita Universidad Autónoma de Puebla (BUAP) and the University of Sonora (UNISON) in Mexico, both CMS collaborators, organised the webinar "The Physics of Elementary Particles", covering topics ranging from LHC experiments to artificial intelligence and quantum computing [16]. Around 130 people attended the webinar. The students expressed a great deal of enthusiasm and appreciation for the organisation of these kinds of events and were eager for more.

Events such as the Virtual Visits and those mentioned above may help educators to deal with this new reality and inspire students. Figure 4 shows university and high school students from different institutions in Mexico at various outreach events related to High Energy Physics.

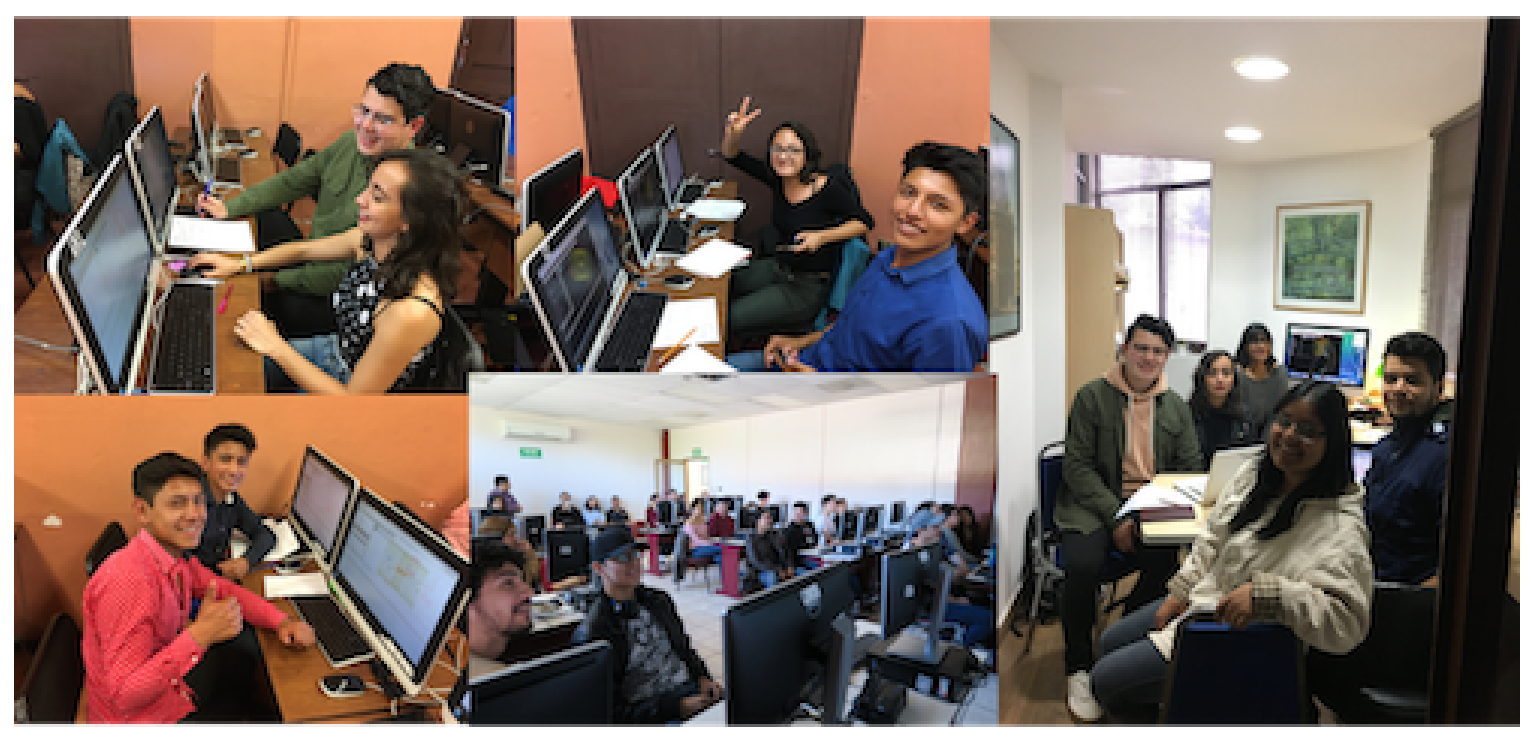

Figure 4: University and high school students attending different CMS outreach events related to High Energy Physics in different institutions in Mexico, such as from the BUAP, Preparatoria Zapata and UNISON. 


\section{Conclusion and Future Directions}

The COVID-19 pandemic has forced society around the world to confront new challenges, including how to teach and share knowledge with students. Outreach events are key to keeping students and the general public engaged with science. During the past years it has been proven that Virtual Visits are one of the most successful forms of outreach to facilitate communication with the public. In September of 2020 Virtual Visits once again resumed, despite the restrictions of the pandemic. These are taking place via Zoom meetings, allowing up to 1,000 participants to connect from home and ask questions through a live chat.

The Virtual Visits teams will continue to pay close attention to the feedback of audiences around the world to improve their experiences, helping teachers at the venues to prepare students for Virtual Visits and providing the necessary materials to increase their interest in STEM topics.

In order to reach a broader audience it is important to continue collaborating with different educational partnerships. The training of new guides at CERN is of particular importance as this will allow us to support the growing demand of Virtual Visits.

As the LHC enters the high luminosity phase, with the expectation of important discoveries to come, Virtual Visits will continue to play an important role connecting the scientific community with audiences worldwide to keep them informed of the latest advances in high energy physics.

\section{Acknowledgements}

We would like to acknowledge the CMS Outreach Group for helping to organise the Virtual Visits and for collecting the statistical data related to them, Marzena Lapka for providing much of the necessary information and material for the preparation of this document, as well as Noemi Beni and Zoltan Szillasi for their important contributions to the technical part of the Virtual Visits both during the preliminary test as well as the visit itself. This work has been supported by project No. A1-S-39980, CB-2017-2018 jointly sponsored by SEP and CONACyT, Mexico.

\section{References}

[1] Higgs discovery (2012) and Nobel prize in physics (2013): http://home.web.cern.ch/topics/higgs-boson, Phys. Lett. B 716 (2012) 1, Phys. Lett. B $716(2012) 30$

[2] The ATLAS Experiment on the Large Hadron Collider at CERN: http://atlas.ch/

[3] The CMS Experiment on the Large Hadron Collider at CERN: https://cms.cern/

[4] CMS Collaboration, The CMS Experiment at the CERN LHC; JINST 3 S08004 (2008)

[5] 200 Irish students at CERN - virtually!: https://cds.cern.ch/record/676868?ln=en

[6] CMS Virtual Visits website: http://cms.cern/interact-with-cms/virtual-visits

[7] Visit CERN: https://visit.cern/welcome 
[8] Hoch, A. \& Alexopoulos, A. (2014). ART@CMS and SCIENCE\&ART@SCHOOL: Novel Education and Communication Channels for Particle Physics. In Astroparticle, Particle, Space Physics and Detectors for Physics Applications-Proceedings of the 14th ICATPP Conference (Vol. 1, pp. 728-736)

[9] CMS open for Virtual Visits, from your school, science festival or exhibition centre https://cylindricalonion.web.cern.ch/blogs/cms-open-virtual-visits-your-schoolscience-festival-or-exhibition-centre

[10] Vidyo: IP-based video conferencing software: http://www.vidyo.com

[11] Hangout with CERN: Reaching the Public with the Collaborative Tools of Social Media, S. Goldfarb, K. Kahle, A. Rao, CHEP 2012, J. Phys.: J.Phys.Conf.Ser. 513 (2014) 062019

[12] CERN Researchers' Night; Bringing CMS to Lithuania, Hungary, Mexico, Italy and Portugal: https://cms.cern/news/bringing-cms-lithuania-hungary-mexico-italy-and-portugal

[13] PoS(EPS-HEP2015)349 ATLAS and CMS Virtual Visits M.Lapka, Digital Communication Awards, hosted by Quadriga University of Applied Sciences: http://www.digital-awards.eu (Award in 2013)

[14] LHC master classes in physics: http://physicsmasterclasses.org/index.php?cat=physics

[15] Big Analysis of Muons in CMS (BAMC) May 2020: https://quarknet.org/content/bamc-may2020-graphics

[16] Webinar "The Physics of Elementary Particles" June 2020, organised by University of Sonora (UNISON) and Benemérita Universidad Autónoma de Puebla (BUAP), Mexico: https://www.youtube.com/watch?v=hyqR4BqoddI 